\title{
Medical Hematology/Oncology Fellows' Perceptions of Online Medical Education During the COVID-19 Pandemic
}

\author{
Eric K. Singhi ${ }^{1}$ (D) Megan M. Dupuis ${ }^{1} \cdot$ Jeremy A. Ross ${ }^{1} \cdot$ Alyssa G. Rieber $^{1} \cdot$ Nishin A. Bhadkamkar ${ }^{1}$
}

Published online: 5 September 2020

(C) American Association for Cancer Education 2020

\begin{abstract}
In response to the COVID-19 social distancing guidelines, residency and fellowship programs transitioned to virtual instruction to deliver didactics and continue with medical education. The efficacy of such a fully online learning environment, however, remains unknown. To investigate its impact on medical education, this study surveyed hematology/oncology fellows at The University of Texas MD Anderson Cancer Center on their attitudes regarding the online-based lecture program. Fellows were emailed a 19-question survey with questions on demographics, ease of technical access to the online platform, level of comfort with participation, knowledge acquisition, wellness, and COVID-19-specific coverage. A free-text question soliciting ways to improve upon online learning was also included. The response rate was $71 \%$ (30/42). Most respondents reported easy/very easy accessibility to the online environment. Seventy-seven percent of the participants did not experience a technical issue. Seventy percent felt comfortable/very comfortable with participating in the conference. Thirty-seven percent felt comfortable/very comfortable with actively offering an answer to questions during the interactive board review session. Eighty-seven percent would have been more willing to offer an answer during the board review session if an anonymous poll format was utilized. Sixty-three percent felt they learned the same amount as they typically do during an in-person session. Thirty-three percent reported they were less focused as compared with an in-person session. One hundred percent of the participants had their questions answered, either at all times $(87 \%)$ or sometimes (13\%). Sixty percent experienced a change in social interactions as compared with an inperson session. Fifty-four percent reported that it was easy/very to balance online attendance despite personal/family commitments. One hundred percent appreciated the flexibility of the online learning environment. Ninety percent felt safer at home attending these lectures compared with receiving these lectures in-person during the COVID-19 pandemic. Overall, most fellows felt comfortable with the transition to a fully online learning environment. Strategies to encourage active participation, enhance social interaction, and provide additional flexibility are still needed.
\end{abstract}

Keywords Oncology $\cdot$ Hematology $\cdot$ Medical education $\cdot$ Online learning $\cdot$ COVID-19 $\cdot$ Medical trainees

\section{Introduction}

The coronavirus disease 2019 (COVID-19) pandemic is leading to changes in residency and fellowship programs nationwide. According to guidance from the Accreditation Council

Previous presentations: None

Electronic supplementary material The online version of this article (https://doi.org/10.1007/s13187-020-01863-6) contains supplementary material, which is available to authorized users.

Eric K. Singhi

eksinghi@mdanderson.org

1 Division of Cancer Medicine, The University of Texas MD Anderson Cancer Center, 1400 Holcombe Blvd., FC11.3055, Houston, TX 77030, USA for Graduate Medical Education (ACGME), training programs must continue to provide adequate resources, training, and supervision, while maintaining work hour requirements [1]. The effects of the COVID-19 pandemic on the didactic curriculum of training programs, as well as the overall psychological effects of the pandemic on trainees in general, remain unknown. At our institution, hematology/oncology fellows have $5 \mathrm{~h}$ of didactic lectures scheduled each week that are protected from other clinical duties. Lectures are taught by key faculty at the University of Texas MD Anderson Cancer Center on a rotating list of topics in hematology and oncology to cover a core curriculum that reviews the biology of benign hematology and malignancies by organ site and associated patient management, while also highlighting the most recent updates in the field. Additional lectures include monthly board review sessions, where fellows review hematology/oncology questions from 
established question banks with a faculty expert available for guidance. Fellows are also required to give one presentation per academic year, which can be an interesting patient case, a journal club presentation, or a research presentation, depending on their year of training.

Under normal conditions, trainees attend these programs in-person weekly, and attendance is tracked using an electronic sign-in mechanism. Due to institutional policies related to social distancing practices as a result of the COVID-19 pandemic, the academic program was transitioned to a fully virtual environment using the video conferencing WebEx software. Fellows are emailed an access link to the online conferencing platform at least $24 \mathrm{~h}$ prior to the scheduled lecture series. Fellows are asked to remain logged in for the entirety of the program. Faculty lecturers are contacted in advance to inform them of the transition to a virtual-only platform and to provide instructions on how to navigate the online conferencing platform, including how to share their PowerPoint presentations, how to utilize the online chat feature, and how to utilize the virtual whiteboard. At a minimum, faculty lecturers are required to deliver their lectures in real time over audio using a built-in microphone on their computers or over the phone. Faculty are also given the option to turn on their webcams during the lecture to offer a more personalized lecture experience. Faculty are additionally encouraged to solicit questions and feedback from the fellows in real time during the lectures, either verbally or using the chat feature on the online conferencing platform. After each lecture, approximately $5 \mathrm{~min}$ are made available for fellows to ask questions to the lecturer, either verbally or using the online group chat feature.

We conducted a survey to assess trainees' attitudes about online learning during the pandemic, the utility of didactic lectures given using an online video conferencing platform, and the effects of these changes on their overall wellness and training experience.

\section{Methods}

This survey-based study was a non-experimental analysis of opinions from a cross-sectional sample of first-, second-, and third-year hematology/oncology fellows at The University of Texas MD Anderson Cancer Center. Fellows were asked about their experiences with an online-based lecture program as compared with an in-person lecture program that was necessitated by the COVID-19 pandemic.

\section{Sample (Table 1)}

An email invitation for participation in the survey was sent to all current hematology/oncology fellows at The University of Texas MD Anderson Cancer Center, as all fellows are required to attend the weekly lecture series. The sample consisted of first-year fellows (13), second-year fellows (15), and third-year fellows (14). The open-access survey link was sent to fellows on March 19, 2020, and one reminder email was sent thereafter. The survey was closed to new respondents on March 24, 2020.

\section{Survey Development}

Survey questions were developed by one investigator (firstyear fellow) and were independently reviewed by 4 physicians. All reviewers have a focus in medical education and were familiar with the format of both lecture models. After revisions following review, two investigators independently tested the survey. It was then uploaded to Google Forms and disseminated to all hematology/oncology fellows by email invitation. The final survey contained 2 questions regarding demographics, 3 questions regarding ease of technical access to the online lecture series, 4 questions regarding level of comfort with aspects of participation, 6 questions comparing the online vs in-person lecture program, and 3 COVID-19specific questions. A free-text question soliciting ways to improve the online-based lecture program was included at the end of the survey. Questions regarding ease of technical access addressed overall ease of access, method of access (phone, web-based application, or computer-based application), and specific technical issues encountered. Questions regarding comfort of participation included overall comfort level, method of participation (group chat, speaking into the microphone), comfort during board review questions, and whether a polling instrument would increase likelihood of participation. Questions that compared the online-based lecture system with the in-person lecture system addressed issues regarding knowledge acquisition, focus, social experience, and flexibility. Questions regarding COVID-19 addressed feelings of safety, satisfaction with COVID-19-specific lectures, and interest in future lectures. The survey is provided in Appendix A.

Table 1 Demographics

\begin{tabular}{lcc}
\hline Characteristic & $N$ & $\%(N)$ \\
\hline Fellowship year & 30 & \\
First & & $37(11)$ \\
Second & & $36(11)$ \\
Third & & $27(8)$ \\
Sex & 30 & \\
Male & & $63(19)$ \\
Female & $30(9)$ \\
Prefer not to say & & $7(2)$ \\
\hline
\end{tabular}




\section{Analysis}

Data was extracted from Google Sheets and was also analyzed by using the Google Forms Summary view. Two independent reviewers assessed the free-text answers to look for themes, and then, answers were categorized based on these themes.

\section{Results}

Of the 42 University of Texas MD Anderson Cancer Center hematology/oncology fellows, 30 (71\%) completed the survey. Participants were $63 \%$ male. Seventy-three percent of participants were first- or second-year fellows, while $27 \%$ of participants were third-year fellows.

Regarding ease of accessibility to the online conferences and use of the WebEx technology, most fellows (77\%) reported that it was easy/very easy to join the WebEx conference (Fig. 1). Ninety-three percent of the participants joined the online WebEx platform using their computer browser, either with computer audio (60\%) or with phone audio (33\%). The majority of participants $(77 \%)$ did not experience a technical issue.

Most fellows (70\%) felt comfortable/very comfortable with participating in the WebEx conference (Fig. 2). When actively participating, $87 \%$ of participants preferred to use the group chat feature, while $3 \%$ preferred to unmute the microphone and speak. When specifically participating in the board review session, $37 \%$ of participants felt comfortable/very comfortable with providing an answer to a question, while $50 \%$ of participants felt neutral and $13 \%$ felt uncomfortable. Eighty-seven percent of participants would have been more willing to offer an answer during the board review session if an anonymous poll format had been utilized.

Focusing on knowledge acquisition, $63 \%$ of participants felt they learned the same amount as they typically do during an in-person lecture session, while $24 \%$ felt that they had learned less (Fig. 3). Thirty-three percent of participants said they were less focused as compared with an in-person lecture session, while $60 \%$ reported they were equally focused. All participants stated they were able to get the answers to their questions, either all of the time $(87 \%)$ or sometimes $(13 \%)$.

With regard to fellows' wellness during the online learning experience, $60 \%$ of participants reported that they experienced a change in social interaction (Fig. 4). Fifty-four percent of participants reported that it was easy/very to balance online attendance from a remote location with personal/family commitments, while $13 \%$ found it to be difficult/very difficult and $33 \%$ were neutral. One hundred percent of the participants appreciated the flexibility of online access to the lecture series.

Given that the COVID-19 pandemic was the driving reason to transition to an online learning environment, the survey also assessed the online learning session's coverage of COVID-19specific lecture material and relevant changes to the fellowship program (Fig. 5). Ninety percent of the participants felt safer remaining at home to attend these lectures as opposed to receiving these lectures in-person during the COVID-19 pandemic. Eighty-four percent of the participants felt satisfied/
Fig. 1 Selected survey questions and responses regarding accessibility of WebEx technology

\section{How difficult was it to join the WebEx conference? 30 responses}

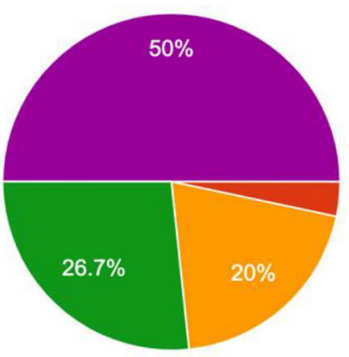

Very difficult

Difficult

Neutral

Easy

Very Easy

5. Did you experience any technical issues (ie network connection difficulties, browser compatibility issues, issues with joining by phone etc.)?

30 responses

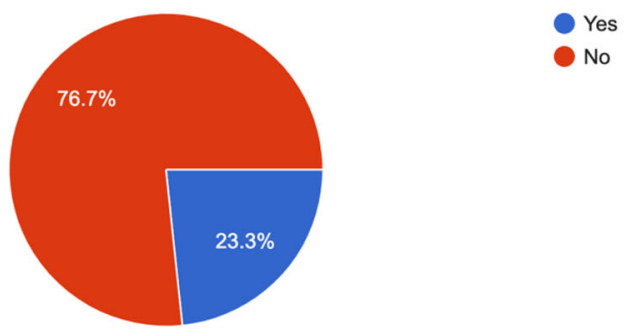


Fig. 2 Selected survey questions and responses regarding participation
6. Overall, how comfortable did you feel with participating in the WebEx conference? 30 responses

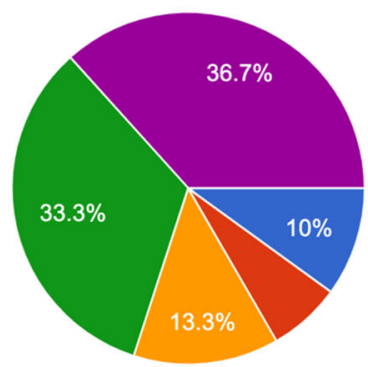

Very uncomfortable

Uncomfortable

Neutral

Comfortable

Very Comfortable

8. How comfortable did you feel with specifically participating in the board review portion of the WebEx conference to offer up an answer?

30 responses
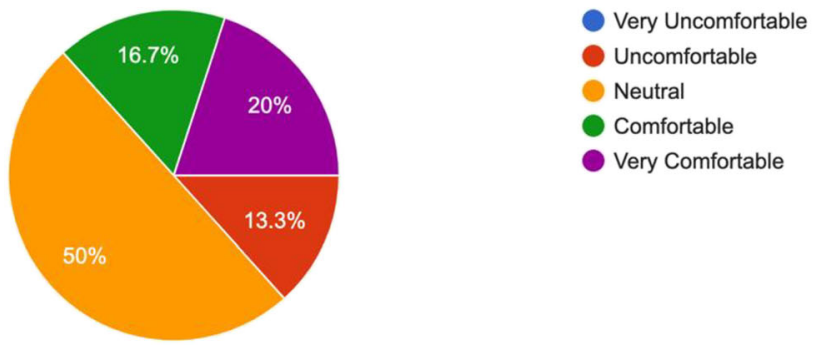

very satisfied with coverage of institution-specific/fellowshipspecific COVID-19 policies.

Review of the open-ended responses demonstrated that several respondents desired the use of an anonymous polling

Fig. 3 Selected survey questions and responses regarding knowledge acquisition
10. With regards to the didactic lectures specific to oncology, rate your knowledge acquisition for online Academic Tuesday (AT) as compared to in-person AT?

30 responses

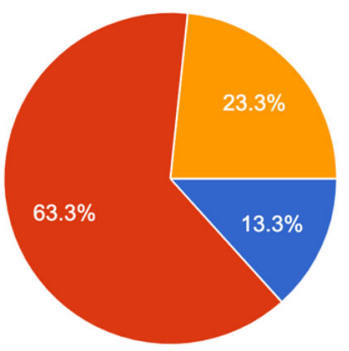

I learned MORE than in-person AT

I learned the SAME amount as inperson AT

I learned LESS than in-person AT
12. Were you able to get answers to your questions?

30 responses

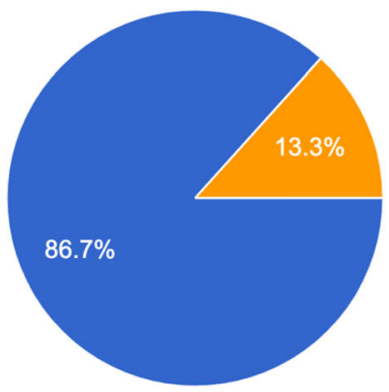

Yes

No

Sometimes 
Fig. 4 Selected survey questions and responses regarding wellness
13. Did you experience a change in social interaction during the online AT as compared to in person AT?

30 responses
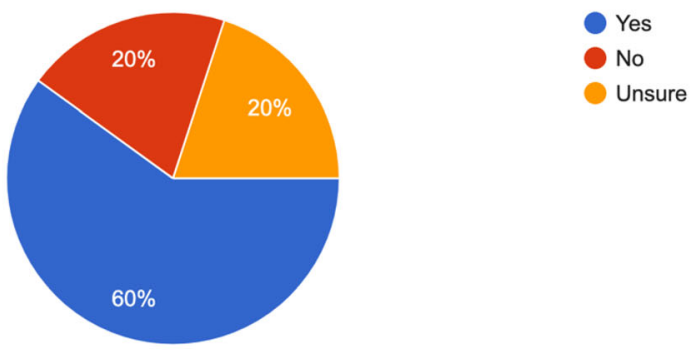

14. How difficult was it to balance online attendance while working remotely from home with personal or family commitments?

30 responses

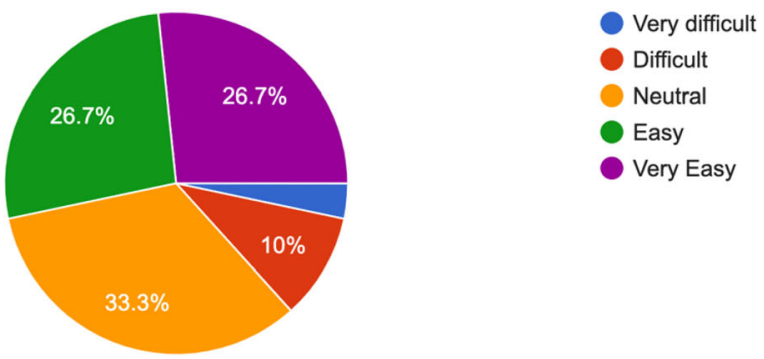

feature to encourage active participation during interactive review sessions.

Looking at the individual survey responses across varying fellowship training levels, $9 \%$ of first-year fellows reported feeling overall uncomfortable/very uncomfortable with the transition to an online lecture series as compared with $21 \%$ second- and third-year fellows. Twenty-seven percent of firstyear fellows reported learning less than normal with the online lecture format, as compared with $21 \%$ of second- and thirdyear fellows. No first-year fellows reported that they had learned more via the online format as compared with inperson format, while $21 \%$ of second- and third-year fellows reported that they had learned more with the online format. Sixty-four percent of first-year fellows reported feeling a change in social interaction with the transition to an onlineonly lecture format as compared with $58 \%$ of second- and third-year fellows.

\section{Discussion}

Our survey aimed to obtain a comprehensive assessment of the online learning experience for fellows by assessing the following core areas: accessibility/use of the online learning environment, participation, knowledge acquisition, wellness, and COVID-19-specific coverage. Overall, our results indicate that the majority of fellows felt comfortable with the
Fig. 5 Selected survey questions and responses regarding COVID19
16. With the restriction of group gatherings during the COVID-19 outbreak, did you feel safer being at home during these lectures?

30 responses
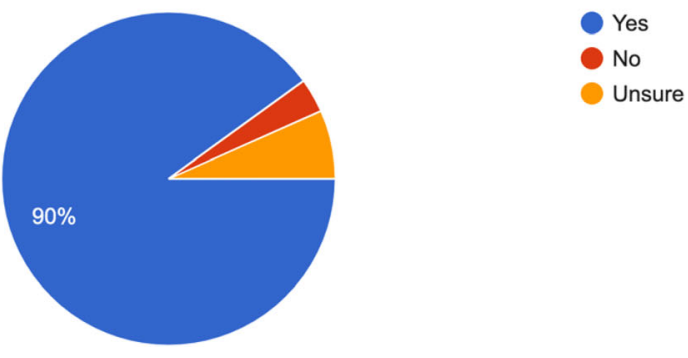
transition to an online learning environment from the traditional in-person learning experience.

When attempting to understand the effect that the online learning experience had on academic and collaborative interactions, only $37 \%$ of participants felt comfortable/very comfortable with providing an answer during the online board review session. The majority of fellows reported that they would have been more willing to offer an answer if an anonymous poll format had been utilized. In doing so, this would offer each fellow a more discrete method to voice their level of comprehension and opinions.

With regard to knowledge acquisition, one third of respondents reported that they were less focused, and nearly a quarter of the participants felt that they had learned less than they would have during an in-person learning experience. In a prior study, researchers conducted a prospective randomized controlled trial to evaluate the level of retention of information in oncology of undergraduate students of physiotherapy who were randomized to complete the course using either a traditional classroom format or an e-learning format [2]. The study found that the level of knowledge acquisition was statistically the same between both learning formats. This is in contrast to the results of our survey, although it is important to note that an objective measure of knowledge acquisition was not incorporated into our survey.

When thinking about how to improve upon the challenge of maintaining our learners' attention, identifying methods to encourage active participation may be a feasible solution. The use of live polls to encourage active participation, collect individual responses, and obtain feedback in real time to foster a larger group discussion may be one such solution. Additionally, utilizing a case-based learning approach to engage fellows in discussion of specific scenarios that resemble typical, real-world patient encounters in the online learning environment may also help to improve upon fellows' motivation and engagement.

Focusing on fellows' wellness so as to enable fellows to thrive both academically and personally throughout their medical training, the majority of fellows reported a change in social and interpersonal interactions as a result of the transition to a full online learning environment. It would be worthwhile to explore how we can further promote the development of social relationships in the online community and how such interactions might influence the online learning process. Additionally, only about half of the participants reported that it was easy/very easy to balance online attendance with personal or family commitments. This is important to keep in mind as many online learners are with limited or no childcare options during the COVID-19 pandemic and, thus, may find it difficult to attend a once weekly, live 5-h online learning session on a regimented schedule. As a solution, assuring that all of the lectures are recorded and that the lecturers' materials are disseminated in a timely manner to all fellows may help to make the learning experience even more flexible for learners with additional personal and family commitments.

Reviewing fellows' survey responses across varying fellowship training levels, there is a suggestion that the level of fellowship training may have an impact on the perception of the online lecture series. Specifically, first-year fellows felt more comfortable overall with the transition to a full online learning lecture series as compared with the second- and thirdyear fellows. This may be due to the fact that the second- and third-year fellows have experienced more of the traditional inperson lectures than the first years and, thus, are likely more accustomed to this lecture format. Additionally, it was interesting to see that no first-year fellows felt that they had learned more through the virtual learning series, while $21 \%$ of secondand third-year fellows reported that they had learned more with the online format.

As a result of the unprecedented COVID-19 pandemic, we acknowledge that the transition to a full online learning environment to deliver didactics was abrupt for our fellowship program, as was the case for most other training programs. However, the primary objective in doing so was to continue with medical education while maintaining our commitment to physical distancing. Although our survey results indicate that overall, most fellows felt comfortable with a transition to an online-only didactic curriculum, previous literature has shown that a pureonline educational approach has not yet been favored over the traditional in-person educational environment [3-5]. A previous study assessed the preferred method of continuing medical education among oncology providers in the state of Florida and found that providers preferred the traditional in-person learning environment over a pure online learning environment [6]. Additionally, the authors showed that providers would prefer that online continuing medical education should supplement and not replace in-person educational interactions.

Thus far, we have responded to feedback from the survey with a goal to improve active participation on behalf of the learners. We have developed a "compendium of cases" lecture where fellows actively discuss patient cases with an expert faculty member that are relevant to the material covered during the online lectures from the month. Additionally, we have incorporated an anonymous polling feature during several of our lectures to encourage active participation. As a future direction, we next plan to evaluate lecturers' and faculty members' perspectives on their transition to the online learning environment, with a particular focus on the impact and quality of the online learning experience.

We acknowledge that our results represent medical trainees' perceptions of online medical education at a single institution; however, it is our hope that by sharing our experience, additional institutions might be inspired to assess their own experience with online education so that we can continue to improve upon the online learning environment during this challenging time. 


\section{Compliance with Ethical Standards}

Conflict of Interest Dr. Eric K. Singhi, Dr. Megan M. Dupuis, and Dr. Jeremy A. Ross are current medical hematology/oncology fellows at The University of Texas MD Anderson Cancer Center.

Ethical Approval Not applicable

\section{References}

1. ACGME response to pandemic crisis. https://www.acgme.org/ covid-19. Accessed March 27, 2020

2. da Costa Vieira RA, Lopes AH, Sarri AJ, Benedetti ZC, de Oliveira CZ (2017) Oncology E-learning for undergraduate. A prospective randomized controlled trial. J Cancer Educ 32:344-351
3. Harris JM Jr, Sklar BM, Amend RW, Novalis-Marine C (2010) The growth, characteristics, and future of online CME. J Contin Educ Heal Prof 30(1):3-10

4. Young KJ, Kim JJ, Yeung G, Sit C, Tobe SW (2011) Physician preferences for accredited online continuing medical education. $\mathrm{J}$ Contin Educ Heal Prof 31(4):241-246

5. Ruiz JG, Mintzer MJ, Leipzig RM et al (2006) The impact of Elearning in medical education. Acad Med 81(3):207-212

6. Cragun D, Besharat AD, Lewis C, Vadaparampil ST, Pal T (2013) Educational needs and preferred methods of learning among Florida practitioners who order genetic testing for hereditary breast and ovarian cancer. J Cancer Educ 28:690-697

Publisher's Note Springer Nature remains neutral with regard to jurisdictional claims in published maps and institutional affiliations. 